\title{
Biotecnologia no Rio de Janeiro: análise de cenário com foco em recursos humanos
}

\author{
Marcia Cristina Paes \\ Tatiane Alves Baptista*
}

\begin{abstract}
RESUMO
O presente artigo trata da política pública de biotecnologia brasileira, tomando como eixo de análise a questão da formação e da capacitação de recursos humanos, especialmente no Estado do Rio de Janeiro. O artigo faz uma análise da biotecnologia de um modo geral, revelando o papel estratégico que os recursos humanos cumprem para o desenvolvimento desse setor. Aborda, ainda, a política implementada no Rio de Janeiro através do Grupo Executivo do Complexo Industrial das Ciências da Vida e a criação do Subgrupo de Trabalho de Recursos Humanos como rede de conhecimento e de formação do Estado.
\end{abstract}

Palavras-chave: Biotecnologia; Educação; Política Pública; Ciência e Tecnologia; Estado do Rio de Janeiro

\begin{abstract}
This article deals with the public policy of the Brazilian biotechnology, taking as a point of analysis the question of the formation and training of human resources, especially in the state of Rio de Janeiro. The article makes an analysis of biotechnology in general, showing the strategic role of human resources fulfills in order to increase the development of this sector. Also addresses the policy implemented in Rio de Janeiro through the Executive Group of the Life Sciences and Industrial Complex and the creation of sub Working Group on Human Resources as a network of knowledge and State formation.
\end{abstract}

Key words: Biotechnology; Education; Science e Technology; State of Rio de Janeiro

\footnotetext{
* Marcia Cristina Paes: Professora associada, Coordenadoria de Estudos Estratégicos e Desenvolvimento (CEED) - Universidade do Estado do Rio de Janeiro. marcia.paes.uerj@gmail.com

Tatiane Alves Baptista: Professora adjunta, Coordenadoria de Estudos Estratégicos e Desenvolvimento (CEED) - Universidade do Estado do Rio de Janeiro. tatianebuerj@gmail.com
} 


\section{A biotecnologia no mundo e no Brasil}

O presente artigo trata da política pública de biotecnologia, especialmente voltada para saúde humana, considerando o seu caráter fundamental para o desenvolvimento do país e do Estado. Para isso recorre a alguns elementos fundamentais do seu processo de surgimento enquanto setor econômico estratégico e situa o Brasil no cenário internacional da pesquisa, oferecendo dados da economia que envolve os produtos biotecnológicos. Diante disso, a reflexão aqui apresentada aponta para o capital humano no processo histórico de conformação da área como um elo indispensável na cadeia de bioprodutos. Mais que isso, o artigo informa que o fortalecimento dos processos formativos de recursos humanos nesta área determina a consolidação da política pública em termos dos resultados esperados.

O acontecimento base da biotecnologia moderna ocorre em 1953 quando Watson e Crick identificaram a estrutura do DNA e, 20 anos depois, esse processo é incrementado com o desenvolvimento da base para a recombinação de segmentos do DNA feita por Stanley Cohen e Herbert Boyer. Este foi um marco definitivo entre a biotecnologia tradicional e a moderna. $\mathrm{O}$ terceiro momento de salto tecnológico nessa área foi atingido quando o programa de pesquisa da biologia molecular foi sacudido pelo escalonamento do sequenciamento genético na segunda parte dos anos 1980.

A partir dos anos 1990, o sequenciamento tornou-se barato e se difundiu globalmente e, com isso, se criaram as condições para que países em desenvolvimento - como Brasil, Índia, Coreia, China e outros - pudessem aperfeiçoar a sua capacidade científica e tecnológica nessa área (Fonseca, 2012).

Atualmente, o país que concentra o maior número de empresas em biotecnologia é a Índia. Na sequência do ranking situam-se a Coreia do Sul, o Canadá, a Alemanha e a China. O Brasil aparece nesse ranking em $11^{\circ}$ lugar (Global Bioeconomy Consulting LLC, 2007), criando novos empregos (cerca de 1 milhão de novos empregos até 2010), desenvolvimento de vacinas e biogenéricos.

De acordo com os dados, a Índia ocupa posição de destaque no cenário da biotecnologia mundial. Dentre os aspectos mais relevantes que contribuem para o posicionamento favorável da Índia, chamamos atenção para a oferta de mão de obra altamente qualificada. Conforme a Agência Brasileira para o Desenvolvimento Industrial, a Índia possui mais de 300 instituições educacionais de alto nível que oferecem cursos em biotecnologia, bioinformática e ciências biológicas, capazes de formar cerca de 500.000 estudantes por ano. 
Possui ainda mais de 100 universidades de Medicina que formam cerca de 17.000 profissionais por ano e mais de 300.000 pós-graduandos nesta área (Global Bioeconomy Consulting LLC, 2007).

A participação do governo em incentivos para este setor é determinante. O Estado contribui para a qualificação da mão de obra, provê infraestrutura laboratorial apropriada para o desenvolvimento das pesquisas, incentivo à criação de incubadoras e parques tecnológicos e à criação de marcos regulatórios que viabilizam a consolidação da biotecnologia em diversas áreas (ABDI, 2008a).

O Brasil começou tardiamente seu processo científico-tecnológico, tendo suas primeiras universidades fundadas apenas a partir do século $\mathrm{XX}$, enquanto universidades europeias e americanas datam, respectivamente dos séculos XI e XVII. Nossos institutos de pesquisa e empresas tecnológicas datam do século XX e nossas financiadoras de pesquisa só foram criadas a partir da década de 1950. A formação de recursos humanos capacitados para Ciência e Tecnologia (C\&T) iniciou-se nos anos 1950, e os últimos 20 anos foram marcados pelo crescente investimento em projetos de diferentes áreas (Fonseca e Uziel, 2012).

O apoio sistemático à biotecnologia moderna no Brasil começou em São Paulo e Minas Gerais, o que fica claro com a criação de redes e parques tecnológicos, e só depois em outros estados, como o Rio de Janeiro e o Rio Grande do Sul. Antes mesmo de o governo federal reconhecer a importância da área de biotecnologia e dirigir investimentos a ela, o Estado de São Paulo criou redes do projeto genoma, um modelo posteriormente adotado pelo governo federal. A Biominas estabeleceu outro modelo de desenvolvimento conhecido como Science Park, também se destacando em relação aos demais estados.

Nos últimos cinco anos houve um investimento crescente do governo federal com a criação de novos editais e de editais conjuntos com os governos estaduais, com a criação de sistemas que visam o maior entrosamento entre universidade e empresa. Alguns grupos nacionais já conduzem linhas de $\mathrm{P} \& \mathrm{D}$ que envolvem empresas nacionais e grupos acadêmicos. Mas são ainda poucos porque o investimento também ainda é insuficiente e, aparentemente, a adesão das empresas também. Como incentivar atividades de alto risco como a P\&D é a grande questão (Fonseca e Uziel, 2012).

O Plano de Ação em C\&T\&I para o Desenvolvimento Nacional - PACTI, cujas ações estão sendo executadas de forma articulada e coordenada por diversos ministérios, tendo à frente o Ministério de Ciência e Tecnologia - MCT, identifica duas áreas de máxima relevância, a biotecnologia e a nanotecnologia, e tem como objetivos (i) fortalecer a gestão e o 
planejamento das atividades governamentais nas áreas de biotecnologia, nanociências e nanotecnologia, de modo a melhor identificar os grandes desafios e as oportunidades para o país; (ii) estabelecer prioridades e criar as condições institucionais, materiais e de recursos humanos para um maior estímulo à inovação por meio da agilização do processo de transferência de conhecimento (ABDI, 2008b).

\subsection{Mercado de biotecnologia no Brasil}

A Lei Federal de Inovação nº 10973/2004 ressalta o papel do Estado em promover e incentivar o desenvolvimento científico e tecnológico do país, conforme os artigos 218 e 219 da Constituição Federal. Quatro anos mais tarde, o Estado do Rio de Janeiro promulga a Lei Estadual de Inovação no 5361/08, regulamentada pelo Decreto no 43302/10.

Pesquisa realizada pela Fundação Biominas em 2007 mostra que Organização para a Cooperação e Desenvolvimento Econômico (OCDE) define uma empresa de biotecnologia como aquela que tem como atividade comercial principal a aplicação tecnológica que utilize organismos vivos, sistemas ou processos biológicos, na pesquisa e desenvolvimento, na manufatura ou na provisão de serviços especializados (Fundação Biominas, 2007).

Um estudo do governo federal em parceria com a Fundação Biominas e a PcW aponta os dados atualizados de 2011 (Fundação Biominas, 2011). No total, identificaram-se 271 empresas de biociências (ou ciências da vida), 143 das quais formam o conjunto de empresas de biotecnologia.

A pesquisa indicou que $82 \%$ das empresas pesquisadas atuam em três áreas principais, a saber: saúde humana (33\%), agronegócios (31\%), insumos (18\%), e deve-se destacar um importante subgrupo de empresas que é formado por micro e pequenas empresas incubadas, as quais por serem nascentes, start-ups, se encontram sem uma situação especial do ponto de vista de suas relações com o mercado, universidades e centros de pesquisa científica, os quais em sua maioria abrigam as incubadoras. Do universo estudado de 143 empresas atuantes em biotecnologia, 25 são incubadas e representam 35,2\% do conjunto total estando concentradas na região Sudeste. De acordo com recente pesquisa publicada (FUNDAÇÃO BIOMINAS, 2011), o setor no Brasil, as empresas de biotec brasileiras ainda estão na sua infância. A maior parte é criada por ação empreendedora de cientistas, sem experiência em negócio e em marketing. Essas empresas estão prioritariamente nas incubadoras de universidades, 
concentrando-se principalmente, na região sudeste do país e dependendo de financiamentos do Estado (FINEP, CNPq e BNDES).

Outro estudo que aborda o desenvolvimento da biotecnologia no Brasil é um artigo recentemente publicado na revista Nature Biotechnology (Rezaie et al., 2008), focalizando especificamente o desenvolvimento de aplicações na área de saúde humana. $\mathrm{Na}$ visão dos autores desse artigo, o desenvolvimento da biotecnologia no Brasil nessa área tem logrado êxito nos últimos anos, com progressos consideráveis e soluções inovadoras.

\section{Recursos humanos na biotecnologia brasileira}

Em todo o mundo, as empresas de biotecnologia, além de pequenas, se localizam e se articulam em forma de rede, procurando se agrupar estrategicamente em parques tecnológicos, em clusters e incubadoras, em geral situadas perto da universidade onde está o capital humano. A condição básica para a existência dessas empresas é o capital humano, seja o conhecimento dos fundadores seja o das pessoas que vão integrar o time daquela empresa. Busca-se mão de obra qualificada (Fonseca, 2012).

Hoje, o Brasil apresenta forte capacitação em material humano, que atua principalmente em pesquisa básica, apresentando e provendo boa formação universitária. $\mathrm{O}$ Brasil tem uma liderança em Pesquisa e Desenvolvimento (P\&D) entre os países em desenvolvimento, apresentando excelentes laboratórios em âmbito federal e estadual, que atuam de forma inteligente formando redes de pesquisa. Produz muita ciência, mas poucos produtos (Fonseca e Uziel, 2012).

Através dos conselhos que regulamentam as profissões, o Comitê Nacional de Biotecnologia buscou verificar a existência de cursos que formem pessoas na área de biotecnologia e muito poucos foram encontrados. Vários contêm o nome, mas, quando se analisa a grade curricular, observa-se que não formam pessoal para biotecnologia. $\mathrm{Na}$ área da escola técnica de nível médio, por exemplo, só foram detectados cinco cursos, sendo o do Rio de Janeiro (no Instituto Federal do Rio de Janeiro - IFRJ) o primeiro. Outros se sucederam nos antigos Centros Federais de Educação Tecnológica e em algumas escolas estaduais do Sistema Paula Souza em São Paulo. Mas são cinco que efetivamente fazem um ensino médio de biotecnologia. Além desse número ser extremamente pequeno, as escolas estão concentradas praticamente na região Sudeste. Seria necessário haver, pelo menos, uma em cada estado. Assim, conclui-se que não estamos formando técnicos de nível médio, em nível 
nacional, em número suficiente para atender à demanda da biotecnologia (Fonseca e Uziel, 2012).

Se analisarmos a formação para a modalidade tecnólogo, é possível identificar no Brasil apenas três cursos. Eles são uma modalidade de ensino de nível superior, com ênfase na formação de pessoal em diferentes áreas de biotecnologia. Analisando os cursos de graduação com formação efetivamente em biotecnologia vamos encontrar quatro. Muitos apresentam nomes sugestivos, como "biociências e biotecnologia" ou "ciências biológicas e biotecnologia", mas o conteúdo não reflete o que o nome do curso propõe. Muitas vezes o termo é utilizado em cursos em que há mais ênfase em biologia molecular ou genética molecular, mas os processos biotecnológicos em si são pouco abordados no ensino de graduação.

\section{Recursos humanos em biotecnologia no Rio de Janeiro}

A Lei Estadual de Inovação dispõe sobre medidas de incentivos à inovação e à pesquisa científica e tecnológica no ambiente produtivo, com vistas à capacitação e ao alcance da autonomia tecnológica, ao desenvolvimento industrial e às inovações de inclusão social no Estado do Rio de Janeiro. A Lei incentiva também as parcerias público-privadas e órgãos do terceiro setor bem como que a política de reestruturação das empresas do governo do Estado crie um ambiente propício para o nascimento de um complexo.

\subsection{Criação do GECIV}

Criado no âmbito do Estado do Rio de Janeiro, o Grupo Executivo do Complexo Industrial das Ciências da Vida do Estado do Rio de Janeiro - GECIV-RJ - tem a finalidade de elaborar, desenvolver, propor e implantar políticas estaduais de fortalecimento do complexo produtivo e de inovação em ciências da vida, bem como propor outras medidas complementares. De acordo com o Decreto 43315/2011, compete ao GECIV-RJ: I - elaborar, desenvolver, propor e implantar políticas estaduais e promover a articulação dos órgãos e entidades do governo estadual, com vistas a viabilizar um ambiente econômico e institucional propício ao desenvolvimento do Complexo Industrial das Ciências da Vida do Estado do Rio de Janeiro; II - constituir grupos de trabalho sobre temas específicos que demandem conhecimento técnico especializado para dar suporte a suas atividades; III - convidar 
instituições acadêmicas de pesquisa e de ensino, profissionais de notório saber na matéria ou especialistas de outros órgãos ou entidades e da sociedade para colaborar com suas atividades; IV - promover a harmonização dos trabalhos realizados pelos grupos de trabalho, comissões e outras instâncias criadas para a implantação dos programas e ações previstos; V - implantar uma rede de cooperação entre nichos tecnológicos no Estado, induzidos inclusive pelo uso de áreas públicas estaduais, que abriguem unidades produtivas inovadoras, rede essa a ser denominada de Complexo Tecnológico da Vida.

O GECIV é composto formalmente por um representante titular e um suplente dos seguintes órgãos do Estado do Rio de Janeiro: Secretaria de Ciência e Tecnologia e suas vinculadas, Agência de Fomento do Estado do Rio de Janeiro - S.A., Companhia de Desenvolvimento Industrial - CODIN, Instituto Vital Brasil S/A, Empresa de Pesquisa Agropecuária do Estado do Rio de Janeiro, Fundação Carlos Chagas Filho de Amparo à Pesquisa - FAPERJ, Fundação BIO-RIO, Rede de Tecnologia e Inovação - REDETEC, Superintendência de Vigilância Sanitária Estadual da Saúde, além das universidades, do SEBRAE, da FIRJAN e de outros órgãos públicos e privados.

Tendo identificado como gargalos em termos da política pública questões relacionadas à regulação, à formação de recursos humanos e à política de financiamento, o GECIV é hoje um articulador do desenvolvimento da biotecnologia no Rio de Janeiro a partir de uma agenda que envolve todos os segmentos da área através de subgrupos de trabalho, organizados em temáticas estratégicas. O Grupo de Trabalho (GT-RH) se inscreve entre eles.

\subsection{Criação do Grupo de Trabalho de Recursos Humanos em Biotecnologia}

Tendo como principal propósito garantir a oferta de profissionais qualificados para atuar na área de biotecnologia e considerando a perspectiva de crescimento do setor de biotecnologia no Estado do Rio de Janeiro, o Grupo Executivo do Complexo Industrial das Ciências da Vida - GECIV/RJ - buscou envolver as principais unidades formadoras da área articuladas no GT-RH.

Composto pela UERJ, UEZO, UENF, UFF, UFRJ, IFRJ, IVB, FIOCRUZ, IORT e UNIFOA, o GT-RH atua hoje no sentido de buscar a fixação dos recursos capacitados na área, incentivando as empresas na valorização do quadro de pessoal, através de uma política de remuneração atrativa e na ampliação da formação profissional, garantido conteúdos de gestão, empreendedorismo, projetos e patentes, além de criar um ambiente integrado propício à 
obtenção de dados atualizados sobre a realidade do mercado de trabalho em biotecnologia, considerando a demanda e a oferta de profissionais, facilitando a fixação de profissionais capacitados junto ao mercado de trabalho.

Desde a sua instalação, o GT-RH do GECIV vem trabalhando na identificação de impasses e na afirmação de estratégias para o fortalecimento do Rio de Janeiro na área de recursos humanos de biotecnologia. Dentre tais estratégias destacam-se: i) aproveitar a capacidade instalada e reaproveitar o capital humano "desatualizado", incentivando as vocações tecnológicas e científicas na área de saúde humana em biotecnologia, do ensino básico à pós-graduação, avaliando seu alcance no sentido de direcionar a abertura de novos âmbitos de formação e capacitação em biotecnologia; ii) capacitar os profissionais nas áreas de planejamento, gestão, regulação e financiamento de empreendimentos em biotecnologia e inovação - no ensino médio, graduação e pós-graduação - integrando conhecimentos teóricos e práticos, com vistas ao mercado de trabalho; iii) formalizar uma coordenação que envolva universidades/unidades de formação, governo e empresários mobilizados para incentivar a formação do capital humano desde o técnico ao pós-graduado; iv) formar banco de dados voltado especialmente para o empreendedor que está iniciando negócio, dando mais viabilidade ao empreendimento e ampliando as oportunidades; v) aprimorar os dados das pesquisas existentes sobre formação e mercado de trabalho, produzindo informações consistentes sobre a demanda e a oferta de profissionais em biotecnologia; vi) discutir ferramentas de apoio às empresas para fixação do profissional através de programas de bolsas, de estágios e de participação acionária; vii) incorporar no âmbito da formação a questão das legislações (nacionais e internacionais), que envolvem o setor de biotecnologia, como a bioética e as patentes no âmbito da propriedade intelectual.

Atualmente o GT-RH tem como principal objetivo mapear a oferta de recursos humanos em biotecnologia por parte das instituições de ensino para atender de forma objetiva e consistente à demanda deste mercado no Estado do Rio de Janeiro. Dessa forma o grupo ganha destacada relevância social por representar um esforço de consolidação da política pública do Estado do Rio de Janeiro para o setor de biotecnologia e, ao mesmo tempo, pelo seu caráter inovador, fortalecendo a comunidade acadêmica através da sua integração.

Trata-se de uma estratégia envolvendo diferentes áreas e atores universitários no sentido de oferecer meios e condições para a interação social, cultural e científica entre os membros da comunidade acadêmica, contribuindo para o compartilhamento e disseminação do saber. 
Ressalta-se que, ao propiciar um ambiente de produção acadêmica que seja sistemático e articulado, dinâmico e complexo, o GT-RH cria condições para o fortalecimento das suas potencialidades intrínsecas contidas em cada elemento da sua composição. Num cenário de isolamento, típico da atividade científica, essa potência encontra-se subsumida, uma vez que os pesquisadores atuam fragmentados em seus núcleos institucionais, enquanto que, no cenário proposto pelo projeto, temos uma mudança significativa, pois, ao atuar de forma compartilhada e integrada, geramos sinergia, alterando a cultura científica do trabalho individualizado, colaborando para a superação da realidade da pouca articulação interna entre os pesquisadores, criando ambientes de integração sistemática, facilitando as trocas acadêmicas e potencializando a capacidade universitária no campo da inovação tecnológica.

Além disso, pode-se dizer que o projeto vem produzindo resultados pragmáticos no sentido da ampliação da capacidade de cada instituição, pois, ao criar as condições materiais para o efetivo compartilhamento do saber através do estabelecimento de um fluxo contínuo de interações acadêmico-institucionais que incluem pessoas, grupos de pesquisa, laboratórios, programas de estudos, entendidos como uma comunidade de interesse comum, captando os caminhos que levam à construção do conhecimento ampliado, o GT-RH reafirma o papel decisivo da academia no processo do desenvolvimento.

\subsection{Cursos de graduação existentes no Estado do Rio de Janeiro}

O Estado do RJ conta com cinco Cursos de Graduação em Biotecnologia, sendo apenas um oferecido por uma instituição privada (UNIFOA).

Todos os cursos encontram-se regulados de acordo com a lei que regulamenta a profissão, oferecendo bacharelado, sendo a UEZO a única instituição a oferecer a modalidade tecnólogo.

Todas as instituições responsáveis estão mostradas na tabela abaixo:

Tabela 1. Unidades formadoras de bacharéis em Biotecnologia no Estado do Rio de Janeiro

\begin{tabular}{|l|l|}
\hline IFRJ & Bacharelado em Ciências Biológicas (Habilitação Biotecnologia) \\
\hline UERJ & Bacharelado em Ciências Biológicas (Habilitação Biotecnologia) \\
\hline UEZO & Bacharelado em Ciências Biológicas \\
\hline UEZO & Tecnólogo em Biotecnologia \\
\hline
\end{tabular}




\begin{tabular}{|l|l|}
\hline UFRJ & Bacharelado em Ciências Biológicas (Habilitação Biotecnologia) \\
\hline UNIFOA & Bacharelado em Ciências Biológicas \\
\hline
\end{tabular}

Fonte: Elaboração própria.

As instituições relacionadas representam grande tradição na área das ciências biológicas de um modo geral, gerando de fato um ambiente facilitado de implementação das matérias de biotecnologia haja vista os equipamentos e laboratórios biomédicos destas instituições e principalmente o quadro de pesquisadores e docentes.

Outro aspecto importante desse quadro é a pós-graduação e a capacidade inerente de pesquisa presente em cada programa. Como sabemos, a área biomédica representa parte significativa do percentual brasileiro de produção de conhecimento e de tecnologias. Isso se deve ao papel da universidade e dos centros de pesquisa, bem como das agências de fomento estaduais e federais.

Acrescentamos a isso a evidente contribuição das incubadoras de empresas, das chamadas "empresas juniores" e das "startups" enquanto estratégias para implantação de uma nova cultura acadêmica, segundo a qual a participação do setor produtivo no processo de construção do conhecimento científico é muito bem-vinda.

Talvez essa seja a grande novidade no quadro geral da política de ensino superior, ciência e tecnologia brasileira, notadamente a desconstrução de velhas verdades do passado, nas quais pairava a ideia de que a presença da empresa levaria à privatização da instituição pública e à perda da autonomia na produção do conhecimento.

Atualmente acompanhamos a implementação de um novo vetor de crescimento da ciência brasileira, desafiada a ampliar seu espectro para além da produção de papers e a assumir, junto com o Estado, uma maior responsabilidade no processo de gestão da saúde pública e da qualidade de vida no país, o que significa incluir na pauta da comunidade científica o apoio ao aparecimento de novas empresas, capazes de levar adiante a produção de biotecnologia no país.

\subsubsection{Perfil dos Cursos de Graduação em Biotecnologia existentes}

Das escolas existentes, duas estão localizadas na zona norte da cidade do Rio de Janeiro (IFRJ e UERJ), uma na zona oeste (UEZO), uma na Baixada Fluminense (UFRJ) e outra no interior do Estado (UNIFOA - Volta Redonda). Todas as escolas foram criadas entre os anos de 2000 e 2011. 
Como pode ser visto na Tabela 2, a duração dos cursos é de quatro anos para todas as universidades, exceto o Curso de Tecnólogo da UEZO que tem a duração de três anos, e a carga horária total varia entre 3.240 horas e 4060 horas. Todos revelam preocupação para o oferecimento de uma formação que atenda o setor produtivo. Cabe ressaltar que os cursos da UERJ e UFRJ ainda não formaram suas primeiras turmas. 
Tabela 2 - Duração e carga horária dos cursos de graduação do Estado do Rio de Janeiro

\begin{tabular}{|c|c|c|c|}
\hline \multicolumn{5}{|c|}{ Duração e Carga Horária dos Cursos } \\
\hline Instituição & Modalidade & Duração do Curso & Carga Horária \\
\hline IFRJ & Graduação & 4 anos & 3.240 horas \\
\hline UERJ & Graduação & 4 anos & 4.055 horas \\
\hline UEZO & Graduação & 4 anos & 4.060 horas \\
\hline UEZO & Tecnólogo & 3 anos & 3.300 horas \\
\hline UFRJ & Graduação & 4 anos & $\begin{array}{c}3.810 \text { horas }+ \\
\text { eletivas }\end{array}$ \\
\hline UNIFOA & Graduação & 4 anos & 3.960 horas \\
\hline
\end{tabular}

Fonte: Elaboração própria.

\subsection{Cursos Técnicos em Biotecnologia existentes no Estado do Rio de Janeiro}

Atualmente existem no Rio de Janeiro três unidades oferecendo Curso de Biotecnologia em nível técnico, duas instituições públicas e uma privada, como mostrado na Tabela 3.

Tabela 3. Unidades formadoras de Técnicos em Biotecnologia no Estado do Rio de Janeiro

\begin{tabular}{|l|l|}
\hline Instituição & Titulação \\
\hline IFRJ & Técnico em Biotecnologia \\
\hline IOC/FIOCRUZ & Técnico em Biotecnologia \\
\hline IORT & Educação Profissional Técnica de Nível Médio \\
\hline
\end{tabular}

Fonte: Elaboração própria.

O IFRJ, antigo CEFET, é uma das mais antigas unidades de formação em nível técnico do país, formando pessoal em biotecnologia desde 1981. Isso significa que há mais de 25 anos o Rio de Janeiro oferece mão de obra de alta qualificação técnica para o setor. Entretanto, não são pouco comuns as afirmações de que há uma preocupante escassez deste tipo de recurso. Essa afirmação muitas vezes surge do próprio segmento empregador.

Há de nossa parte a hipótese de que esse capital humano, exatamente por receber uma formação generalista e de excelência, acaba sendo atraído por condições mais competitivas 
em termos salariais para outras áreas da economia fluminense, tais como o setor químico e o de óleo e gás.

Esse ponto merece destaque, pois ultrapassa o limite de atuação e de capacidade efetiva de atuação da política pública na realidade do mercado de trabalho em biotecnologia, uma vez que recai sobre a lógica intrínseca de funcionamento das leis da produção. É preciso que o próprio setor empresarial demandante esteja sensível a essa realidade de competição, valorizando o profissional demandado.

\subsubsection{Perfil dos Cursos Técnicos em Biotecnologia existentes}

Todas as escolas participantes estão localizadas na cidade do Rio de Janeiro, sendo duas na zona norte (IFRJ e IOC/FIOCRUZ) e uma na zona sul (IORT). Essa informação reitera a tendência de concentração das oportunidades na capital fluminense. Vale indagar sobre a pertinência ou não da criação de oportunidades no interior do Estado, estratégia praticada com sucesso pelo Estado de São Paulo, por exemplo.

Como informado acima, o primeiro Curso Técnico de Biotecnologia do ERJ data do ano de 1981 e a criação do mais recente data do ano 2000. Para ingressar nesses cursos notamos que tanto o IFRJ quanto o IORT aceitam o aluno egresso do ensino fundamental, enquanto o IOC/FIOCRUZ recebe alunos oriundos do ensino médio.

Como pode ser visto na Tabela 4, os cursos técnicos duram entre dois e quatro anos, enquanto a carga horária total está entre 2025 e 5020 horas. Dado importante da formação se refere ao fato de que todos os cursos exigem estágio supervisionado, com carga horária entre 220 e 480 horas, e todos revelam preocupação para o oferecimento de uma formação que atenda o setor produtivo. Isso significa que esse aluno entra em contato com as empresas e laboratórios desde o início do seu processo de formação, abrindo as unidades formadoras para uma interação direta com o mercado.

Tabela 4. Duração e carga horária dos Cursos Técnicos em Biotecnologia do Estado do Rio de Janeiro

\begin{tabular}{|l|l|l|l|}
\hline Instituição & Modalidade & Duração do curso & Carga Horária \\
\hline IFRJ & Técnico & 4 anos & 4.287 horas \\
\hline IOC/FIOCRUZ & Técnico & 2 anos & $\begin{array}{l}1.845 \mathrm{~h} \mathrm{+} \mathrm{360h} \mathrm{de} \\
\text { Estágio Obrigatório }\end{array}$ \\
\hline IORT & Técnico & 3 anos & 5.020 horas \\
\hline
\end{tabular}

Fonte: Elaboração própria. 
Vislumbramos aqui um grande potencial para atividades científicas inovadoras em termos da produção de novas tecnologias em produtos biológicos, pois o momento do estágio supervisionado abrange o processo formativo concomitante com o processo da bioprospecção, através do qual o aluno poderá experimentar a metodologia tradicional em termos da sua formação e ao mesmo tempo confrontar esse conhecimento com as novas tecnologias para fins industriais.

Podemos dizer que, embora o oferecimento de cursos especificamente na área de biotecnologia ainda seja reduzido em comparação com outros estados de outros países como a Índia, por exemplo, quando consideramos esse mesmo quadro observando o oferecimento de cursos nas áreas afins, tais como ciências farmacêuticas, engenharia química, química, biofísica, medicina veterinária, bioquímica etc, notamos um cenário diferenciado em termos de oportunidades em recursos humanos capacitados na área.

\section{Nota conclusiva}

Embora a situação brasileira no cenário mundial da produção de conhecimento e da produção industrial em biotecnologia ainda esteja muito aquém da sua real capacidade, isto é, ainda esteja muito focada na produção acadêmica de papers e ainda possa avançar mais em patentes e bioprodutos aplicados e com resultados de mercado, facilitando a abertura de novos empreendimentos em biotecnologia no Brasil, podemos afirmar que os últimos três anos foram marcados por uma progressiva articulação política e institucional que em curto prazo gerou importantes processos no sentido do amadurecimento deste setor no Brasil.

Particularmente o Estado do Rio foi capaz de estabelecer de maneira integrada uma nova política pública, rompendo com paradigmas da forma tradicional de fazer política, uma vez que reuniu numa única perspectiva o setor público, o setor privado e as universidades e instituições de ensino.

Alguns resultados deste trabalho podem ser percebidos na atração de novas empresas para o estado, na criação e aprovação pela Agência Nacional de Vigilância Sanitária ANVISA - de uma frente para otimização do registro e controle dos biotecnológicos em parceria com o GECIV, no surgimento de novos editais da FAPERJ incentivando a produção de biotecnologia e inovação nas empresas e na ação coordenada e integrada das principais instituições de formação e capacitação no estado gerando estudos e alternativas para o 
atendimento da demanda emergente de profissionais, que, sem perder a formação generalista, estejam atentos e sintonizados com as novas determinações do mundo produtivo.

Tudo isso resulta de uma rotina de trabalho e investimentos institucionais que, a partir de uma metodologia estabelecida no âmbito do GECIV e dos seus subgrupos de trabalho, vem desenhando um quadro cada vez mais bem definido do Cluster de Biotecnologia do Estado do Rio de Janeiro.

\section{Referências bibliográficas}

ABDI. Biotecnologia: Iniciativa Nacional de Inovação - Panorama da Biotecnologia no Mundo e no Brasil. 2008a. Disponível em: http://www.abdi.com.br/Estudo/Panorama\%20Setorial\%20Biotecnologia.pdf

. Biotecnologia: Iniciativa Nacional de Inovação - Estudo Prospectivo. Visão de Futuro e Agenda INI Biotecnologia 2008-2025. 2008b. Disponível em: http://www.abdi.com.br/Estudo/Estudo\%20Prospectivo\%20Biotecnologia.pdf

FONSECA, M. G. D. Identificando tendências de mercado e oportunidades em biotecnologia. In: UZIEL, D. (org.). Biotecnologia no Brasil: financiamento, parcerias e desafios. Rio de Janeiro: EdUerj, 2012. pp. 29-44.

FONSECA, M. G. D. e UZIEL, D. A história e o momento atual da biotecnologia no Brasil. In: UZIEL, D. (org.). Biotecnologia no Brasil: financiamento, parcerias e desafios. Rio de Janeiro: EdUerj, 2012. pp. 17-28.

FUNDAÇÃO BIOMINAS. Estudo de Empresas de Biotecnologia do Brasil. Belo Horizonte: Fundação Biominas, 2007. Disponível em: http://win.biominas.org.br/estudobio/estudo/. Acesso em outubro de 2008.

A indústria de Biociências Nacional: Caminhos para o Desenvolvimento. 2011. Disponível em: http://www.biominas.org.br/conteudo.php?idicod=1\&paccod=15

GLOBAL BIOECONOMY CONSULTING LLC. Global Hubs and Global Nodes of Biotecnology: an international scan of biotechnology strategies, initiatives and institutional capacity. 3. ed. Washington: Global Bioeconomy Consulting LLC, Ago 2007. pp. 85-92. 
REZAIE, R. et al. Brazilian health biotech: fostering crosstalk between public and private sectors. Nature Biotechnology. Vol. 26, n. 6, jun. 2008. 